Published in final edited form as:

J Pain. 2017 October ; 18(10): 1229-1236. doi:10.1016/j.jpain.2017.05.007.

\title{
Depression and Pain in Asian Americans and Whites with Knee Osteoarthritis
}

\author{
Hyochol Ahn, $\mathrm{PhD}^{1}$, Michael Weaver, $\mathrm{PhD}^{2}$, Debra Lyon, $\mathrm{PhD}^{2}$, Eunyoung Choi, $\mathbf{R N}^{1}$, and \\ Roger B. Fillingim, $\mathrm{PhD}^{3}$ \\ ${ }^{1}$ The University of Texas Health Science Center at Houston School of Nursing, Houston, Texas \\ 2University of Florida College of Nursing, Gainesville, Florida \\ ${ }^{3}$ University of Florida Pain Research and Intervention Center of Excellence, Gainesville, Florida
}

\begin{abstract}
Few studies have examined the underlying psychosocial mechanisms of pain in Asian Americans. Using the biopsychosocial model, we sought to determine whether variations in depression contribute to racial group differences in symptomatic knee osteoarthritis pain between Asian Americans and non-Hispanic whites. The sample consisted of 100 participants, including 50 Asian Americans (28 Korean Americans, 9 Chinese Americans, 7 Japanese Americans, 5 Filipino Americans, and 1 Indian American) and 50 age- and sex-matched non-Hispanic whites with symptomatic knee osteoarthritis pain. The Centers for Epidemiologic Studies Depression Scale was used to assess symptoms of depression, and the Western Ontario and McMaster Universities Osteoarthritis Index and the Graded Chronic Pain Scale were used to measure clinical pain. In addition, quantitative sensory testing was used to measure experimental sensitivity to heat- and mechanically-induced pain. The results indicated that higher levels of depression in Asian Americans may contribute to greater clinical pain and experimental pain sensitivity. These findings add to the growing literature regarding ethnic and racial differences in pain and its associated psychological conditions, and additional research is warranted to strengthen these findings.

Perspective-This article demonstrates the contribution of depression to clinical pain and experimental pain sensitivity in Asian Americans with knee osteoarthritis. Our results suggest that Asian Americans have higher levels of depressive symptoms and that depression plays a relevant role in greater clinical pain and experimental pain sensitivity in Asian Americans.
\end{abstract}

\section{Keywords}

Asian Americans; depression; pain; quantitative sensory testing; osteoarthritis

Corresponding Author: Hyochol Ahn, PhD, APRN, ANP-BC, Department of Nursing Systems, School of Nursing, The University of Texas Health Science Center at Houston, 6901 Bertner Avenue, Ste. 539A, Houston, TX 77030. Tel: (713) 500-2179. Fax: (713) 500-0266. Hyochol.Ahn@uth.tmc.edu.

The authors hold no conflicts of interest.

Publisher's Disclaimer: This is a PDF file of an unedited manuscript that has been accepted for publication. As a service to our customers we are providing this early version of the manuscript. The manuscript will undergo copyediting, typesetting, and review of the resulting proof before it is published in its final citable form. Please note that during the production process errors may be discovered which could affect the content, and all legal disclaimers that apply to the journal pertain. 
Chronic pain affects 100 million people in the United States and accounts for annual costs of up to $\$ 635$ billion. ${ }^{23,30}$ Arthritis is one of the leading causes of pain and disability in people 45 years and older, ${ }^{28,33}$ and about half of the 53 million adults diagnosed with arthritis reported trouble performing their activities of daily living due to arthritis pain. ${ }^{7}$ Knee osteoarthritis (OA) is the most common of the arthritic conditions, ${ }^{19,} 31,33$ and racial/ethnic group differences in knee OA prevalence and its adverse effects have been widely documented. ${ }^{3}$ Indeed, some evidence indicates that knee OA prevalence is $16-75 \%$ higher in Asian females than age-matched white females. ${ }^{3,}$, 20, 60

While it is generally believed that Asian Americans' sensitivity to pain is comparable to or lower than that of non-Hispanic whites (NHWs), ${ }^{29}$ recent studies reported that Asian Americans had higher levels of pain. ${ }^{2}, 15,16$ Indeed, a handful of studies in healthy young adults have reported greater experimental pain sensitivity in Asians compared to NHWs ${ }^{27,}$ 32, 36, 47 In addition, older Asian Americans with knee OA were shown to have greater clinical and experimental pain than NHWs. ${ }^{1}$ Even though Asian American was the fastest growing ethnic group in the United States between 2000 and 2010, ${ }^{53}$ most studies have not included significant numbers of Asian American participants, and therefore, little is known about Asian Americans' pain experiences.

Moreover, Asian Americans experience severe depressive symptoms as they deal with crosscultural stresses. ${ }^{38,58}$ These negative psychological factors may lead to higher levels of pain in Asian Americans. However, few studies have examined the underlying psychosocial mechanisms of pain in Asian Americans and the contribution of these biopsychosocial factors to ethnic group differences in OA pain experiences.

The biopsychosocial model of pain hypothesizes that pain is influenced by biological factors (e.g., age, sex), psychological factors (e.g., depression), and sociocultural factors (e.g., race/ ethnicity). ${ }^{21}$ Using this model, we sought to determine whether variations in depression contribute to racial group differences in symptomatic knee OA pain between Asian Americans and age- and sex-matched NHWs. We hypothesized that high levels of depression contribute to clinical and experimental pain and that depression mediates the relationship between race and knee pain.

\section{Methods}

\section{Study participants}

Detailed selection criteria and enrollment procedures were published has been described previously. ${ }^{2}$ Fifty Asian American participants with knee OA pain were recruited via posted fliers and an email advertisement sent to Asian community listservs in North Central Florida between June 2014 and October 2014, and 50 sex- and age-matched NHW individuals were randomly selected from a previous study conducted between January 2010 and October 2013, Understanding Pain and Limitations in Osteoarthritic Disease (UPLOAD). Four age groups ( 45 to 55 years, 56 to 65 years, 66 to 75 years, and 76 to 85 years) were used for age matching. For both groups, individuals were included if they were 45-85 years old and had self-reported unilateral or bilateral knee OA pain. Asian Americans were considered eligible if they were Asian American by self-report and could speak and read English. Individuals 
were excluded if they had a prosthetic knee replacement; had a serious medical illness, such as uncontrolled hypertension (blood pressure > 150/95), heart failure, or a history of acute myocardial infarction; had peripheral neuropathy; had a systemic rheumatic disorder such as rheumatoid arthritis, systemic lupus erythematosus, or fibromyalgia; used opioids daily; had cognitive impairment (<23/30 on the Mini-Mental Status Examination); or had been hospitalized within the preceding year for psychiatric illness.

All procedures were approved by the Institutional Review Board of the affiliated university prior to commencement. After informed consent was obtained, the study participants completed a general health and demographic questionnaire, including age, sex, education level, height, weight, and employment status. For Asian Americans, we collected acculturation level using Suinn-Lew Asian Self Identity Acculturation scale (range 1-5, with higher scores indicating more acculturation to Western culture $)^{48}$ and nativity or place of birth. The principal investigator and research data collector were of Asian American descent.

\section{Self-reported measures of depression and clinical pain}

The Centers for Epidemiologic Studies Depression Scale (CES-D) ${ }^{8}$ was used to assess symptoms of depression. The CES-D consists of 20 items that measure the frequency of depressive symptoms during the past week on a 4-point Likert scale that ranges from 0 (rarely or none of the time) to 3 (most or all of the time). The total score of the CES-D (range 0-60, with more depressive symptoms) was used in this study. The CES-D has been studied over several years in various contexts and patient populations including Asian Americans. ${ }^{27}$

The Western Ontario and McMaster Universities Osteoarthritis Index (WOMAC) ${ }^{9}$ pain subscale was used to assess pain symptoms of knee OA. The WOMAC pain subscale consists of 5 items that measure the severity of symptoms of arthritis pain in the past 48 hours using a 5-point Likert scale that ranges from 0 (no pain) to 4 (extreme pain). The total score of the WOMAC Pain (range 0 -20, with higher scores indicating more severe symptoms of arthritis pain) was used in this study. The Graded Chronic Pain Scale (GCPS) ${ }^{54}$ was used to measure global pain intensity and interference with activities. The GCPS consists of 3 items that measure current pain intensity and the worst and average pain intensity as well as 3 items that measure the degree to which the respondents' knee pain interfered with their daily activities during the past 6 months using a numeric scale ranging from 0 (no pain/interference) to 10 (pain as bad as could be/extreme change). These items were averaged and multiplied by 10 to generate a pain score and a disability score. Total score of GCPS Pain (range 0 -100, with higher scores indicating higher pain intensity during the past 6 months) and GCPS Disability (range $0-100$, with higher scores indicating more severe interference with daily activities during the past 6 months) were used in this study.

\section{Quantitative sensory testing procedures}

Thermal testing procedures-All thermal stimuli were delivered using a computercontrolled Medoc Pathway neurosensory analyzer. Heat pain thresholds and heat pain tolerance were assessed on both the index knee and the ipsilateral ventral forearm using an ascending method of limits. The position of the thermode was moved among 3 sites between 
trials to avoid sensitization and/or habituation of cutaneous receptors. From a baseline of $32^{\circ} \mathrm{C}$, the thermode temperature increased at a rate of $0.5^{\circ} \mathrm{C}$ per second until the participants responded by pressing a button on a handheld device. Participants were instructed to press the button when the sensation "first becomes painful" to assess the heat pain threshold and when they "no longer feel able to tolerate the pain" to assess their heat pain tolerance. The results of the 3 individual trials were averaged to generate an overall heat pain threshold temperature and heat pain tolerance temperature, which were used for analysis. We assessed both heat pain threshold and tolerance, as threshold is thought to reflect the sensorydiscriminative component of pain, while tolerance is thought to reflect the affectivemotivational dimension.

Mechanical testing procedures-We assessed two types of mechanical pain responses. Pressure pain thresholds (PPTs) assessed sensitivity to blunt mechanical pressure applied to deep tissues (i.e. muscle and joint). In addition, we used nylon monofilaments to assess cutaneous mechanical sensitivity to punctate stimuli.

PPTs were evaluated using a handheld Medoc digital pressure algometer (Algomed). A constant rate of $30 \mathrm{kPa}$ per second was applied to measure the pressure pain threshold at 5 sites: the medial aspect of the index knee, lateral aspect of the index knee, ipsilateral quadriceps, trapezius, and dorsal forearm. The order of the testing sites was counterbalanced and randomized. Participants were instructed to press the button when the sensation "first becomes painful" to assess the pressure pain threshold. The results of the 3 trials at each body site were averaged to generate pressure pain threshold at each site, and then, these pressure pain thresholds at 5 sites were averaged to derive an overall measure of pressure pain threshold.

Following the assessment of the pressure pain threshold, participants underwent a procedure to assess their sensitivity to punctate mechanical stimuli. A calibrated nylon monofilament delivering a target force of 300 grams was applied on both the index patella and the back of the ipsilateral hand to obtain verbal ratings of the pain intensity on a scale of 0 (no pain sensation) to 100 (the most intense pain sensation imaginable) following 10 contacts at a rate of 1 contact per second. The procedure was repeated twice, and the ratings were averaged to generate an overall punctate pain sensitivity.

\section{Statistical analyses}

Data were examined for missing and out-of-range values using descriptive statistics appropriate for the level of measurement. To form composite measures of quantitative sensory testing measures, $\mathrm{z}$-scores were computed for heat pain threshold and heat pain tolerance measurements at the arm and knee; pressure pain threshold measurements at the medial knee, lateral knee, quadriceps, trapezius, and dorsal forearm; and punctate pain measurements at the patella and hand. Then, those z-scores for each pain measure were averaged across body sites to derive an overall measure of heat pain threshold, heat pain tolerance, pressure pain threshold, and punctate pain for use in the analyses.

Separate path analytic models (Figure 1) were estimated to evaluate indirect effects (mediation) for ethnicity (coded 0 for NHW and 1 for Asian American) operating through 
depression on each of seven measures of clinical and experimental pain. The path models enabled the testing of both direct and indirect effects. Model fit, path coefficient estimates, and 95\% highest posterior density (HPD) credibility intervals (CIs) for parameter estimates were produced using the Bayesian estimation method in Mplus, version 7.4. 5, 40 Bayesian estimation was chosen because of its advantages over and superior performance relative to frequentist estimation methods (e.g., maximum likelihood), especially when used with relatively small samples. Specific advantages include (a) better parameter estimates; (b) better accuracy (in terms of stability and coverage rate) for confidence (credibility in Bayesian terms) intervals, even in the presence of non-normal distributions such as occur with mediation effects; ${ }^{11,56}$ and (c) more reliable small-sample model-rejection performance than likelihood-ratio tests. ${ }^{4}$ HPD credibility intervals contain the highest posterior density. ${ }^{24,40}$ Model fit was evaluated using criteria and methods recommended by Muthén and colleagues. ${ }^{39}$ Path coefficients with a 95\% CI that did not include the value 0 were considered significant.

\section{Results}

\section{Participants}

A total of 100 participants (50 Asian Americans and 50 NHWs) were enrolled in the study. The Asian American group included 28 Korean Americans, 9 Chinese Americans, 7 Japanese Americans, 5 Filipino Americans, and 1 Indian American. Forty-six participants (92\%) were born in Asian Countries, and only $4(8 \%)$ were born in the United States. On average, Asian Americans had lived in the United States for about $21 \pm 15$ years, and they were mildly acculturated to the Western culture (Suinn-Lew Asian Self Identity Acculturation score $=2.18 \pm 0.55$ ).

The two ethnic groups were similar in terms of sex (62\% females in each), age (mean $[\mathrm{M}]=$ 55 years, standard deviation $[\mathrm{SD}]=8$ in both groups), and employment status (70\% of Asian Americans and $62 \%$ of NHWs were employed). NHW participants had a higher body mass index than Asian Americans (NHWs: $\mathrm{M}=28.63, \mathrm{SD}=6.05$; Asian Americans: $\mathrm{M}=24.16$, $\mathrm{SD}=3.03, P<.001$ ), and a lower proportion of NHWs had graduate degrees (NHW: $18 \%$; Asian: 40\%; overall: likelihood ratio $\chi^{2}=10.45, \mathrm{DF}=4 ; P=0.03$; cell $\chi^{2}=2.09$ ). Descriptive statistics for variables used in the path models appear in Table 1, and the augmented correlation matrix for model variables is provided in Table 2. A majority of the latter bivariate correlations could be qualitatively characterized as weak or smaller. Given the sample size, absolute values for correlations would need to be greater than .26 to achieve statistical significance at the two-tailed .01 level.

From a theoretical perspective, there may be a feedback loop between depressive symptoms and pain, with pain influencing depressive symptoms and depressive symptoms in turn influencing pain. ${ }^{21}$ Within a path analytic framework, that would produce a non-recursive relationship between CES-D and Pain. Non-recursive models were examined for each of the pain outcomes, but none converged due to collinearity introduced by the non-recursive path. 


\section{Model results}

Fit for each of the models was acceptable, with all 95\% CIs for the difference between observed and replicated $\chi^{2}$ values encompassing 0 , all posterior predictive values $>.45$, and convergence of posterior parameter trace plots. Table 3 provides results from the path analyses, including direct and indirect effects and the 95\% HPD credibility intervals for each of the pain measure models. The direct effect for race on pain outcome represents the difference in mean between Asian and non-Hispanic whites after controlling for depression, and the direct effect for depression on pain represents the effect of depression on pain after controlling for race. The indirect effect represents the effect of race on pain operating through depression. In each of the models, the direct effect for race on depression indicated that Asian Americans had higher mean CES-D scores than NHWs (difference in means = 6.7; 95\% CI: 3.31, 10.31).

\section{Direct effect of race on pain measures after controlling for depression}

There were significant direct effects for race on pain measures after controlling for depression. The direct effect of race on the GCPS pain score $(c=8.26)$ and punctate pain score $(c=0.90)$ indicated that Asian American participants had higher mean scores on those pain measures than NHWs after controlling for CES-D scores. Based on the direct effect of race on the heat pain threshold $(c=-1.21)$, heat pain tolerance $(c=-1.17)$, and pressure pain threshold $(c=-0.91)$, Asian Americans demonstrated lower mean scores on those experimental pain measures than NHWs after controlling for CES-D scores. After controlling for CES-D score, Asian American and Non-Hispanic Whites were found to have similar means for WOMAC Pain and GCPS Disability.

\section{Direct and indirect effect of depression on pain measures}

Depression exhibited positive direct effects, and race exhibited positive indirect effects through CES-D scores on the WOMAC pain score $\left(b=0.20, c^{\prime}=1.29\right)$, GCPS pain score $(b=$ $\left.0.50, \mathrm{c}^{\prime}=3.23\right)$, GCPS disability score $\left(\mathrm{b}=0.78, \mathrm{c}^{\prime}=5.09\right)$, and punctate pain score $(\mathrm{b}=$ $0.03, c^{\prime}=0.18$ ). These results indicate that participants with higher CES-D scores tended to have higher values on those pain measures after controlling for race. Considering that race had no direct effects on the WOMAC pain score or GCPS disability score but had indirect effects through CES-D scores, race effects were likely fully mediated through CES-D scores for those measures. Also, given the direct effects for race on the GCPS pain and punctate pain scores, along with the indirect effects of race on those measures operating through CES-D scores, race effects on those measures were partially mediated through CES-D scores.

In addition, depression exhibited negative direct effects, and race exhibited negative indirect effects through CES-D scores on heat pain tolerance $\left(b=-0.02, c^{\prime}=-0.13\right)$. Those effects indicate that, after controlling for CES-D, Asian American participants had lower mean heat pain tolerance than NHWs, participants with higher CES-D scores tended to have lower heat pain tolerance, and race effects on heat pain tolerance were partially mediated through CESD scores. However, we did not find direct effects for depression on the heat pain threshold or pressure pain threshold after controlling for race. 


\section{Discussion}

Using the biopsychosocial model of pain, ${ }^{21}$ we sought to determine whether variations in depression mediated racial group differences in clinical pain and experimental pain sensitivity between Asian Americans and age- and sex-matched NHWs. Asian Americans have been understudied in pain research, possibly owing to the misconception that Asian Americans are stoic and less sensitive to pain, as previous studies of racial or ethnic group differences in pain have largely focused on African Americans. ${ }^{13}$ The results of our mediational analysis suggest that higher levels of depression in Asian Americans may contribute to their higher levels of clinical pain and experimental pain sensitivity; however, the mediating effects of race through depression were more consistently observed for clinical pain measures.

Our results are consistent with previous findings showing that Asians have more severe depressive symptoms than whites. For example, Young and colleagues ${ }^{58}$ reported that Asian American college students exhibited significantly higher levels of depression than whites, which is similar to the findings reported by Okazaki. ${ }^{41}$ Similarly, Williams and colleagues ${ }^{57}$ found that healthy Asian adults had higher depressive symptoms than white adults in the United Kingdom. One possible explanation is that Asian Americans may develop crosscultural stresses from conflicting Asian and American cultural norms, and these stresses may then lead to more severe depressive symptoms. ${ }^{25,} 38$ Moreover, a previous study showed that Asian Americans were less likely than European Americans to seek social support for managing stress. ${ }^{50}$ Cross-cultural stresses and a lack of social support may interact to increase pain. These two factors could interact to increase pain and depression, as social support represents an important buffer against stress ${ }^{35}, 46$; hence, the combination of acculturation stress and low social support may be particularly potent. In addition, the impact of acculturation stress and low social support may be magnified among Asian Americans with comorbid pain and depression; both are risk factors for pain and depression, $34,37,45,59$ which can, in turn, be mutually exacerbating. Moreover, cultural traditions that emphasize stoicism (e.g., Confucianism) may prevent Asian Americans from seeking adequate treatment for pain and depression; consequently, the severity of their pain and depressive symptoms may increase. ${ }^{27,52}$ Collectively, our results extend the associations among these factors to the subgroup of older Asian Americans with knee OA.

Our results are also consistent with previous findings in which depressive symptoms were associated with clinical pain and experimental pain sensitivity. For example, Axford and colleagues ${ }^{6}$ reported that increased depression was associated with greater clinical pain in 170 persons with knee OA. Moreover, Euteneuer and colleagues ${ }^{18}$ reported that 37 outpatients with depression had greater experimental pain sensitivity than 48 healthy controls. In contrast, a recent meta-analysis found that depressed patients had higher experimental pain thresholds than controls, while pain tolerance was not different. ${ }^{51}$ However, it is important to note that patients in our study generally showed low to moderate depressive symptoms and would not meet the criteria for a depressive disorder. Our study extends these findings and has addressed the contribution of depression to ethnic group differences in clinical pain and experimental pain sensitivity between Asian Americans and NHWs. In this study, depression was not significantly associated with experimental pain 
thresholds (heat pain threshold and pressure pain threshold) after controlling for race, which may be a result of having an insufficient sample size to detect those differences. It is also interesting to note that racial/ethnic concordance between researchers and study participants may contribute to levels of expressed pain. Hsieh et al. (2011) found that Chinese participants exposed to Chinese researchers reported higher affective pain and more nonverbal pain behavior than Chinese participants exposed to Euro-Canadian researchers. ${ }^{26}$

Pain and depression share multiple, putative biological pathways, yet the biological mechanisms for the intersection of pain and depression are not yet clear. ${ }^{44}$ Shared molecular mechanisms underlying the relationship between depression and pain could include monoamine neurotransmitters such as norepinephrine, serotonin, and dopamine; substance $\mathrm{P}$ and corticotrophin-releasing factor ${ }^{14}$; and amino acid neurotransmitters including $\gamma$ aminobutyric acid and glutamate. ${ }^{10}$ Similar alterations in the sympathoadrenal and hypothalamo-pituitary-adrenal axis may also contribute to the comorbidity of pain and depression. ${ }^{12}$ Further, elevated levels of proinflammatory cytokines, such as tumor necrosis factor- $a$ and interleukin-6, may contribute to both pain and depression. ${ }^{55}$ Recently, the endogenous cannabinoid system was implicated in reciprocal interactions between depression and pain. ${ }^{22}$

Our findings should be interpreted in light of our study's limitations. First, we included a small sample of Asian Americans and were unable to examine differences within different Asian subgroups. Although our sample included individuals from multiple Asian countries, most participants were Korean. Thus, our sample was somewhat homogenous and not fully representative of the Asian American population, which limits the generalizability of the study findings. Second, we measured self-reported depressive symptoms using the CES-D and did not assess the underlying mechanisms of higher levels of depression in Asian Americans. However, we can speculate that Asian Americans may develop cross-cultural psychological distress and have higher depressive symptoms. Third, owing to the crosssectional nature of our study, the causality and directionality of associations were derived solely from theoretical predictions and cannot be empirically established. Indeed, it could be argued that greater pain contributes to higher depression levels in Asian Americans. Fourth, we included patients with self-reported knee OA pain without radiographic examination. This radiographic information would strengthen the interpretation and generalizability of these results; however, some literature shows that radiographic findings have not been a strong predictor of clinical symptoms such as pain. ${ }^{43,49}$ Finally, we did not include other potential factors related to pain, such as anxiety, fatigue, or sleep problems, and, due to collinearity, we were unable to model the feedback loop between depression and pain.

Given these caveats, the results from this study could have important clinical implications after further replication. Our findings support the need for increased attention to the assessment and treatment of depressive symptoms in Asian Americans in the management of their pain.Importantly, given the cultural traditions that emphasize Asians' stoicism, ${ }^{27}$ health care providers may need to more systematically assess pain and depression among their Asian American patients, as these patients may be less likely to report these symptoms. Moreover, understanding the factors that may contribute to depressive symptoms in Asian Americans experiencing pain could lead to improved treatment of both pain and depressive 
symptoms. For example, if acculturation stress is found to influence depressive and/or pain symptoms among Asian Americans, then culturally sensitive stress management interventions would be indicated. ${ }^{17,38,42}$ Similarly, to mitigate the impact of low social support on these comorbid symptoms, clinicians should encourage patients to enhance social networks through communication skills and valued social activities.

The findings from this study also provide a foundation for future research. Also, comprehensive psychological and sociocultural variables as well as radiographic examination of OA severity prior to study entry are needed to generalize these findings. First, future studies with larger samples of Asian Americans are needed to validate the findings of these path analyses. Larger samples would provide for a more sensitive test of model fit, as well as allow the testing of structural equation models incorporating measurement models and non-recursive relationships. Larger samples and more complex models would also allow for the inclusion of comprehensive psychological and sociocultural variables, and the inclusion of radiographic measures of OA severity are also needed to generalize these findings. Second, additional research is needed to identify barriers to pain and depression management in Asian Americans with knee OA pain. Future studies should assess Asian Americans' interactions with healthcare providers, particularly those from nonconcordant ethnic groups to whom Asian Americans may be reluctant to describe the severity of their symptoms.

Few studies have examined the link between pain and depression in Asian Americans, the fastest growing ethnic minority group in the United States. Our results suggest that depression plays a relevant role in the racial and ethnic group differences in pain between Asian Americans and NHWs and add to the body of literature regarding ethnic and racial differences in pain and its associated psychological conditions. Future longitudinal randomized controlled trials with rigorous depression treatment methodologies and adequate numbers of Asian Americans are needed to determine the clinical implications of these findings.

\section{Acknowledgments}

The authors would like to thank the staff at the University of Florida Pain Research and Intervention Center of Excellence for their work on this project.

Disclosures: This study was funded by the University of Florida Clinical and Translational Science Institute Clinical Research Pilot Project Award, supported in part by the NIH/NCATS Clinical and Translational Science Award to the University of Florida (UL1 TR000064) and by NIH/NIA grants R37AG033906 and K07AG046371. The funding agencies had no role in the study design, methods, data collection and analysis, or preparation of the manuscript.

\section{References}

1. Ahn H, Fillingim R, Lyon D, Garvan C, Choi E. Experimental pain sensitivity and clinical pain severity in Asian Americans compared to non-Hispanic whites with knee osteoarthritis. J Pain. 2015; 16:S31.

2. Ahn H, Weaver M, Lyon D, Kim J, Choi E, Staud R, Fillingim RB. Differences in clinical pain and experimental pain sensitivity between Asian Americans and Whites with knee osteoarthritis. Clin J Pain. 2016; 33:174-180. 
3. Allen KD. Racial and ethnic disparities in osteoarthritis phenotypes. Curr Opin Rheumatol. 2010; 22:528-532. [PubMed: 20473172]

4. Asparouhov T, Muthén B. Bayesian analysis of latent variable models using Mplus Version 4. 2010

5. Asparouhov, T., Muthén, B. [Accessed May 10, 2017] Bayesian analysis using Mplus: Technical implementation. Available at:http://www.statmodel.com/download/Bayes3.pdf

6. Axford J, Heron C, Ross F, Victor CR. Management of knee osteoarthritis in primary care: pain and depression are the major obstacles. J Psychosom Res. 2008; 64:461-467. [PubMed: 18440398]

7. Barbour KE, Helmick CG, Theis KA, Murphy LB, Hootman JM, Brady TJ, Cheng YJ. Prevalence of doctor-diagnosed arthritis and arthritis-attributable activity limitation-United States, 2010-2012. Morb Mortal Wkly Rep. 2013; 62:869-873.

8. Beekman AT, Deeg DJ, Van Limbeek J, Braam AW, De Vries MZ, Van Tilburg W. Criterion validity of the Center for Epidemiologic Studies Depression scale (CES-D): results from a community-based sample of older subjects in The Netherlands. Psychol Med. 1997; 27:231-235. [PubMed: 9122304]

9. Bellamy N, Buchanan WW, Goldsmith CH, Campbell J, Stitt LW. Validation study of WOMAC: a health status instrument for measuring clinically important patient relevant outcomes to antirheumatic drug therapy in patients with osteoarthritis of the hip or knee. J Rheumatol. 1988; 15:1833-1840. [PubMed: 3068365]

10. Benson C, Mifflin K, Kerr B, Jesudasan SJ, Dursun S, Baker G. Biogenic Amines and the Amino Acids GABA and Glutamate: Relationships with Pain and Depression. Mod Trends Pharmacopsychiatri. 2015; 30:67-79.

11. Biesanz JC, Falk CF, Savalei V. Assessing Mediational Models: Testing and interval estimation for indirect effects. Multivariate Behav Res. 2010; 45:661-701. [PubMed: 26735714]

12. Blackburn-Munro G. Hypothalamo-pituitary-adrenal axis dysfunction as a contributory factor to chronic pain and depression. Curr Pain Headache Rep. 2004; 8:116-124. [PubMed: 14980146]

13. Campbell CM, France CR, Robinson ME, Logan HL, Geffken GR, Fillingim RB. Ethnic differences in diffuse noxious inhibitory controls. J Pain. 2008; 9:759-766. [PubMed: 18482870]

14. Campbell LC, Clauw DJ, Keefe FJ. Persistent pain and depression: a biopsychosocial perspective. Biol Psychiatry. 2003; 54:399-409. [PubMed: 12893114]

15. Chan MY, Hamamura T, Janschewitz K. Ethnic differences in physical pain sensitivity: role of acculturation. Pain. 2013; 154:119-123. [PubMed: 23149393]

16. Dhingra L, Lam K, Homel P, Chen J, Chang VT, Zhou J, Chan S, Lam WL, Portenoy R. Pain in underserved community-dwelling Chinese American cancer patients: demographic and medical correlates. Oncologist. 2011; 16:523-533. [PubMed: 21402591]

17. Edrington J, Sun A, Wong C, Dodd M, Padilla G, Paul S, Miaskowski C. A pilot study of relationships among pain characteristics, mood disturbances, and acculturation in a community sample of Chinese American patients with cancer. Oncol Nurs Forum. 2010; 37:172-181. [PubMed: 20189922]

18. Euteneuer F, Schwarz MJ, Hennings A, Riemer S, Stapf T, Selberdinger V, Rief W. Depression, cytokines and experimental pain: evidence for sex-related association patterns. J Affect Disord. 2011; 131:143-149. [PubMed: 21167607]

19. Felson DT, Lawrence RC, Dieppe PA, Hirsch R, Helmick CG, Jordan JM, Kington RS, Lane NE, Nevitt MC, Zhang Y, Sowers M, McAlindon T, Spector TD, Poole AR, Yanovski SZ, Ateshian G, Sharma L, Buckwalter JA, Brandt KD, Fries JF. Osteoarthritis: new insights. Part 1: the disease and its risk factors. Ann Intern Med. 2000; 133:635-646. [PubMed: 11033593]

20. Felson DT, Nevitt MC, Zhang Y, Aliabadi P, Baumer B, Gale D, Li W, Yu W, Xu L. High prevalence of lateral knee osteoarthritis in Beijing Chinese compared with Framingham Caucasian subjects. Arthritis Rheum. 2002; 46:1217-1222. [PubMed: 12115226]

21. Fillingim RB. Individual differences in pain responses. Curr Rheumatol Rep. 2005; 7:342-347. [PubMed: 16174481]

22. Fitzgibbon M, Finn DP, Roche M. High Times for Painful Blues: The endocannabinoid system in pain-depression comorbidity. Int J Neuropsychopharmacol. 2016; 19:pyv095.

23. Gaskin DJ, Richard P. The economic costs of pain in the United States. J Pain. 2012; 13:715-724. [PubMed: 22607834] 
24. Gelman, A., Carlin, JB., Stern, HS., Rubin, DB. Bayesian data analysis. 2. New York: Chapman \& Hall; 2004.

25. Hovey JD, Kim SE, Seligman LD. The influences of cultural values, ethnic identity, and language use on the mental health of Korean American college students. J Psychol. 2006; 140:499-511. [PubMed: 17066754]

26. Hsieh AY, Tripp DA, Ji LJ. The influence of ethnic concordance and discordance on verbal reports and nonverbal behaviours of pain. Pain. 2011; 152:2016-2022. [PubMed: 21616598]

27. Hsieh AY, Tripp DA, Ji LJ, Sullivan MJ. Comparisons of catastrophizing, pain attitudes, and coldpressor pain experience between Chinese and European Canadian young adults. J Pain. 2010; 11:1187-1194. [PubMed: 20452836]

28. Hunter DJ, McDougall JJ, Keefe FJ. The symptoms of osteoarthritis and the genesis of pain. Rheum Dis Clin North Am. 2008; 34:623-643. [PubMed: 18687276]

29. Im EO, Chee W, Guevara E, Liu Y, Lim HJ, Tsai HM, Clark M, Bender M, Suk Kim K, Hee Kim Y, Shin H. Gender and ethnic differences in cancer pain experience: a multiethnic survey in the United States. Nurs Res. 2007; 56:296-306. [PubMed: 17846550]

30. Institute of Medicine. Relieving pain in American: a blueprint for transforming prevention, care, education, and research. Washington, DC: National Acadmies Press; 2011.

31. Jordan JM, Helmick CG, Renner JB, Luta G, Dragomir AD, Woodard J, Fang F, Schwartz TA, Abbate LM, Callahan LF, Kalsbeek WD, Hochberg MC. Prevalence of knee symptoms and radiographic and symptomatic knee osteoarthritis in African Americans and Caucasians: the Johnston County Osteoarthritis Project. J Rheumatol. 2007; 34:172-180. [PubMed: 17216685]

32. Komiyama O, Wang K, Svensson P, Arendt-Nielsen L, Kawara M, De Laat A. Ethnic differences regarding sensory, pain, and reflex responses in the trigeminal region. Clin Neurophysiol. 2009; 120:384-389. [PubMed: 19110468]

33. Lawrence RC, Felson DT, Helmick CG, Arnold LM, Choi H, Deyo RA, Gabriel S, Hirsch R, Hochberg MC, Hunder GG, Jordan JM, Katz JN, Kremers HM, Wolfe F. Estimates of the prevalence of arthritis and other rheumatic conditions in the United States. Part II Arthritis Rheum. 2008; 58:26-35. [PubMed: 18163497]

34. LeMaster JW, Broadbridge CL, Lumley MA, Arnetz JE, Arfken C, Fetters MD, Jamil H, Pole N, Arnetz BB. Acculturation and post-migration psychological symptoms among Iraqi refugees: a path analysis. Am J Orthopsychiatry. 2017 Mar 2. [Epub ahead of print].

35. Li LW, Liang J. Social exchanges and subjective well-being among older Chinese: does age make a difference? Psychol Aging. 2007; 22:386-391. [PubMed: 17563194]

36. Lu Q, Zeltzer L, Tsao J. Multiethnic differences in responses to laboratory pain stimuli among children. Health Psychol. 2013; 32:905-914. [PubMed: 23668844]

37. Maneze D, Salamonson Y, Poudel C, DiGiacomo M, Everett B, Davidson PM. Health-seeking behaviors of Filipino migrants in Australia: the influence of persisting acculturative stress and depression. J Immigr Minor Health. 2016; 18:779-786. [PubMed: 26050239]

38. Mui AC, Kang SY. Acculturation stress and depression among Asian immigrant elders. Soc Work. 2006; 51:243-255. [PubMed: 17076122]

39. Muthén B, Asparouhov T. Bayesian SEM: A more flexible representation of substantive theory. Psychol Methods. 2012; 17:313-335. [PubMed: 22962886]

40. Muthén, LK., Muthén, BO. Mplus user's guide. 7. Los Angeles: CA, Muthén \& Muthén; 1998-2005.

41. Okazaki S. Sources of ethnic differences between Asian American and white American college students on measures of depression and social anxiety. J Abnorm Psychol. 1997; 106:52-60. [PubMed: 9103717]

42. Palmer B, Macfarlane G, Afzal C, Esmail A, Silman A, Lunt M. Acculturation and the prevalence of pain amongst South Asian minority ethnic groups in the UK. Rheumatology (Oxford). 2007; 46:1009-1014. [PubMed: 17401133]

43. Pells JJ, Shelby RA, Keefe FJ, Dixon KE, Blumenthal JA, Lacaille L, Tucker JM, Schmitt D, Caldwell DS, Kraus VB. Arthritis self-efficacy and self-efficacy for resisting eating: relationships to pain, disability, and eating behavior in overweight and obese individuals with osteoarthritic knee pain. Pain. 2008; 136:340-347. [PubMed: 17764844] 
44. Pilowsky I, Chapman CR, Bonica JJ. Pain, depression, and illness behavior in a pain clinic population. Pain. 1977; 4:183-192. [PubMed: 600541]

45. Riley JL 3rd, Gibson E, Zsembik BA, Duncan RP, Gilbert GH, Heft MW. Acculturation and orofacial pain among Hispanic adults. J Pain. 2008; 9:750-758. [PubMed: 18456564]

46. Roberts MH, Klatzkin RR, Mechlin B. Social support attenuates physiological stress responses and experimental pain sensitivity to cold pressor pain. Behav Med. 2015; 49:557-569.

47. Rowell LN, Mechlin B, Ji E, Addamo M, Girdler SS. Asians differ from non-Hispanic Whites in experimental pain sensitivity. Eur J Pain. 2011; 15:764-771. [PubMed: 21561793]

48. Suinn RM, Ahuna C, Khoom G. The Suinn-Lew Asian self-identity acculturation scale: concurrent and factorial validation. Educ Psychol Meas. 1992; 52:1046-1051.

49. Szebenyi B, Hollander AP, Dieppe P, Quilty B, Duddy J, Clarke S, Kirwan JR. Associations between pain, function, and radiographic features in osteoarthritis of the knee. Arthritis Rheum. 2006; 54:230-235. [PubMed: 16385522]

50. Taylor SE, Sherman DK, Kim HS, Jarcho J, Takagi K, Dunagan MS. Culture and social support: who seeks it and why? J Pers Soc Psychol. 2004; 87:354-362. [PubMed: 15382985]

51. Thompson T, Correll CU, Gallop K, Vancampfort D, Stubbs B. Is pain perception altered in people with depression? A systematic review and meta-analysis of experimental pain research. J Pain. 2016; 17:1257-1272. [PubMed: 27589910]

52. Tung WC, Li Z. Pain beliefs and behaviors among Chinese. Home Health Care Manag Prac. 2015; 27:95-97.

53. United States Census Bureau. [Accessed May 1, 2017] The Asian population: 2010 census briefs. Available at: https://www.census.gov/prod/cen2010/briefs/c2010br-11.pdf

54. Von Korff M, Ormel J, Keefe FJ, Dworkin SF. Grading the severity of chronic pain. Pain. 1992; 50:133-149. [PubMed: 1408309]

55. Walker AK, Kavelaars A, Heijnen CJ, Dantzer R. Neuroinflammation and comorbidity of pain and depression. Pharmacol Rev. 2014; 66:80-101. [PubMed: 24335193]

56. Wang L, Preacher KJ. Moderated mediation analysis using Bayesian methods. Struct Equ Modeling. 2015; 22:249-263.

57. Williams ED, Nazroo JY, Kooner JS, Steptoe A. Subgroup differences in psychosocial factors relating to coronary heart disease in the UK South Asian population. J Psychosom Res. 2010; 69:379-387. [PubMed: 20846539]

58. Young CB, Fang DZ, Zisook S. Depression in Asian-American and Caucasian undergraduate students. J Affect Disord. 2010; 125:379-382. [PubMed: 20303181]

59. Zeiders KH, Umana-Taylor AJ, Updegraff KA, Jahromi LB. Acculturative and enculturative stress, depressive symptoms, and maternal warmth: examining within-person relations among Mexicanorigin adolescent mothers. Dev Psychopathol. 2015; 27:293-308. [PubMed: 25004391]

60. Zhang Y, Xu L, Nevitt MC, Aliabadi P, Yu W, Qin M, Lui LY, Felson DT. Comparison of the prevalence of knee osteoarthritis between the elderly Chinese population in Beijing and whites in the United States: The Beijing Osteoarthritis Study. Arthritis Rheum. 2001; 44:2065-2071.

[PubMed: 11592368] 


\section{Highlights}

- Asian Americans have higher levels of depression and pain than non-Hispanic whites.

- Higher levels of depression in Asian Americans contribute to their higher levels of clinical pain.

- Higher levels of depression in Asian Americans contribute to their higher levels of experimental pain sensitivity. 


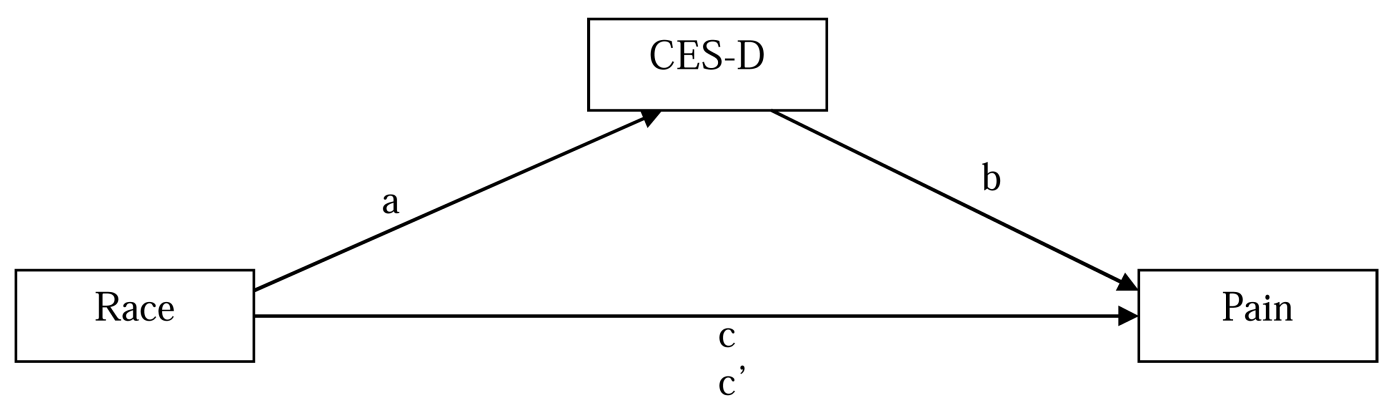

Figure 1.

Mediation model. CES-D = Centers for Epidemiologic Studies Depression Scale. a = direct effect of race on CES-D; $b=$ direct effect of CES-D on pain measures after controlling for race; $c=$ direct effect of race on pain measures after controlling for CES-D. $c^{\prime}=$ indirect effect of race on pain measures operating through CES-D. For pain measures (dependent variables), we measured on the WOMAC Pain (range 0 -20, with higher scores indicating more severe symptoms of arthritis pain), GCPS Pain (range 0 -100, with higher scores indicating higher pain intensity during the past 6 months), and GCPS Disability (range 0 -100 , with higher scores indicating more severe interference with daily activities during the past 6 months). To produce composite quantitative sensory testing measures, average zscores were computed for heat pain threshold and heat pain tolerance measurements at the arm and knee; pressure pain threshold measurements at the medial knee, lateral knee, quadriceps, trapezius, and dorsal forearm; and punctate pain measurements at the patella and hand. 
Table 1

Descriptive Statistics for depressive and pain-related characteristics among Asian American and Non-Hispanic White participants $(\mathbf{N}=100)$

\begin{tabular}{lcc}
\hline Variable, Mean (SD) & NHW $(\mathbf{n}=\mathbf{5 0})$ & Asian $(\mathbf{n}=\mathbf{5 0})$ \\
\hline WOMAC Pain & $5.32(3.48)$ & $7.44(3.59)$ \\
GCPS Pain & $39.67(15.78)$ & $51.33(18.02)$ \\
GCPS Disability & $30.46(19.53)$ & $39.27(22.86)$ \\
Heat Pain Threshold ${ }^{\dagger}$ & $0.62(0.61)$ & $-0.62(0.81)$ \\
Heat Pain Tolerance ${ }^{\dagger}$ & $0.65(0.49)$ & $-0.65(0.91)$ \\
Pressure Pain Threshold ${ }^{\dagger}$ & $0.51(0.94)$ & $-0.51(0.48)$ \\
Punctate Pain ${ }^{\dagger}$ & $-0.55(0.63)$ & $0.55(0.91)$ \\
CES-D & $8.56(8.73)$ & $15.34(8.73)$ \\
\hline
\end{tabular}

Note.

${ }^{\dagger}$ Average z-score; SD = standard deviation; NHW = non-Hispanic white; CES-D = Centers for Epidemiologic Studies Depression Scale. Measured on the CES-D total scores range from 0 - 60, with higher scores indicating more severe depressive symptomatology; WOMAC = Western Ontario and McMaster Universities Osteoarthritis Index. Measured on the WOMAC Pain, total scores range from 0 -20, with higher scores indicating more severe symptoms of arthritis pain; GCPS = Graded Chronic Pain Scale. Measured on the GCPS Pain, total scores range from 0 -100, with higher scores indicating higher pain intensity during the past 6 months. Measured on the GCPS Disability, total scores range from $0-100$, with higher scores indicating more severe interference with daily activities during the past 6 months. To produce composite quantitative sensory testing measures, average z-scores were computed for heat pain threshold and heat pain tolerance measurements at the arm and knee; pressure pain threshold measurements at the medial knee, lateral knee, quadriceps, trapezius, and dorsal forearm; and punctate pain measurements at the patella and hand. As we have previously reported, ${ }^{2}$ significant group differences emerged for each of these variables. 
Table 3

Results of Bayesian analysis: estimated direct and indirect effects with $95 \%$ credibility interval $(\mathbf{N}=\mathbf{1 0 0})$

\begin{tabular}{llll}
\hline Pain Variables $\left(\mathbf{R}^{2}\right)$ & $\mathbf{c}(\mathbf{9 5 \%} \mathbf{C I})$ & $\mathbf{b}(\mathbf{9 5 \%} \mathbf{C I})$ & $\mathbf{c}^{\prime}(\mathbf{9 5 \%} \mathbf{C I})$ \\
\hline WOMAC Pain $(0.30)$ & $0.79(-0.57,1.94)$ & $0.20(0.12,0.27)$ & $1.29(0.51,2.19)$ \\
GCPS Pain $(0.17)$ & $8.26(1.13,15.28)$ & $0.50(0.12,0.88)$ & $3.23(0.64,7.02)$ \\
GCPS Disability $(0.15)$ & $3.48(-5.29,12.12)$ & $0.78(0.31,1.26)$ & $5.09(1.61,10.14)$ \\
Heat Pain Threshold ${ }^{\dagger}(0.42)$ & $-1.21(-1.53,-0.91)$ & $-0.01(-0.02,0.01)$ & $-0.03(-0.16,0.09)$ \\
Heat Pain Tolerance $^{\dagger}(0.47)$ & $-1.17(-1.48,-0.87)$ & $-0.02(-0.04, .-0.00)$ & $-0.13(-0.28,-0.01)$ \\
PPT $^{\dagger}(0.33)$ & $-0.91(-1.24,-0.60)$ & $-0.02(-0.03,0.00)$ & $-0.10(-0.24,0.02)$ \\
Punctate Pain $^{\dagger}(0.38)$ & $0.90(0.57,1.22)$ & $0.03(0.01,0.05)$ & $0.18(0.04,0.35)$ \\
\hline
\end{tabular}

NOTE:

${ }^{\dagger}$ Average z-score; CI = credibility interval; WOMAC = Western Ontario and McMaster Universities Osteoarthritis Index; GCPS = Graded Chronic Pain Scale; PPT = pressure pain threshold. $\mathrm{c}=$ direct effect of race on pain measures after controlling for the Centers for Epidemiologic Studies Depression Scale (CES-D); b = direct effect of CES-D score on pain measures after controlling for race; $c^{\prime}=$ indirect effect of race on pain measures operating through CES-D scores. 